\title{
El rol del apoyo social percibido en la predicción de la inteligencia emocional entre adolescentes de la República Dominicana
}

\author{
Mercedes Nancy Jiménez, Inge Axpe, e Igor Esnaola \\ Universidad del País Vasco (España)
}

\begin{abstract}
En el presente estudio se analiza la capacidad predictiva del apoyo social percibido (personas significativas, familia y amistades) sobre la inteligencia emocional considerada desde el modelo de habilidad (atención, claridad y reparación) y desde el modelo mixto (intrapersonal, interpersonal, manejo del estrés y adaptabilidad), en función del sexo y la edad. Participaron 490 estudiantes de educación secundaria, 300 chicas $(61.2 \%)$ y 190 chicos $(38.8 \%)$, subdivididos en dos grupos de edad, de 11 a 14 años $(M=12.49$, $D T=.615)$ y de 15 a 19 años $(M=15.06, D T=.811)$ residentes en la República Dominicana. Para medir el apoyo social se ha utilizado el cuestionario Multidimensional Scale of Perceived Social Support (MSPSS) y para medir la inteligencia emocional el Trait Meta Mood Scale (TMMS) y el Emotional Quotient Inventory: Youth Version Short (EQ-i: YV-S). En general, los resultados muestran que las diferentes fuentes del apoyo social predicen la inteligencia emocional entre las chicas, y solamente el apoyo familiar predice la inteligencia emocional entre los chicos. Estos datos apuntan hacia la necesidad de favorecer el apoyo social percibido en la adolescencia, especialmente entre las mujeres, por su mayor relación con la IE, especialmente, con la IE habilidad.
\end{abstract}

Palabras clave: Apoyo de personas significativas, apoyo familiar, apoyo de las amistades, inteligencia emocional, adolescencia.

The role of perceived social support in predicting emotional intelligence among adolescents in the Dominican Republic. The present study analyses the predictive capacity of perceived social support (significant people, family and friends) on emotional intelligence, considered from the skill model (attention, clarity and repair) and from the mixed model (intrapersonal, interpersonal, stress management, and adaptability), according to gender and age. Participants were 490 high school students, 300 girls $(61.2 \%)$ and 190 boys (38.8\%), subdivided into two age groups, 11 to 14 years $(M=12.49, S D=.615)$ and 15 to 19 years $(M=15.06, S D=.811)$ resident in the Dominican Republic. The Multidimensional Scale of Perceived Social Support (MSPSS) questionnaire was used to measure social support and the Trait Meta Mood Scale (TMMS) and the Emotional Quotient Inventory: Youth Version Short (EQ-i: YV-S) were used to measure emotional intelligence. In general, the results show that different sources of social support predict emotional intelligence among girls, and only family support predicts emotional intelligence among boys. These data point to the need to promote perceived social support in adolescence, especially among women, due to its greater relationship with EI, especially with EI ability.

Keywords: Support of significant people, family support, support from friends, emotional intelligence, adolescence.

Correspondencia: Mercedes Nancy Jiménez. Departamento de Psicología Evolutiva y de la Educación. Universidad del País Vasco/Euskal Herriko Unibertsitatea (UPV/EHU). C/ Tolosa Hiribidea $\mathrm{n}^{\mathrm{o}}$ 70, C.P. 20018. Donostia, Guipuzkoa (España). E-mail: mercedesnancyimenez@gmail.com 
La adolescencia es uno de los periodos más complicados del ciclo vital debido a los múltiples cambios que ocurren a nivel físico, intelectual, emocional y social; siendo, en este último caso, patente la mayor necesidad de aceptación social, afecto e identificación con los pares (Salavera, Usán, y Teruel, 2019). Precisamente, el apoyo social y la inteligencia emocional (IE) son considerados elementos claves en el éxito de las personas, tanto en el ámbito personal, social, como académico (Pérez-Escoda, Filella, Alegre, y Bisquerra, 2018). El apoyo social se define como el conjunto de provisiones instrumentales y/o expresivas, reales, o percibidas, aportadas por la comunidad, las redes sociales y las amistades, que pueden producirse tanto en situaciones cotidianas como de crisis (Lin y Ensel, 1989). Los estudios concernientes al apoyo social tienden a diferenciar entre el apoyo social recibido, referido a las características de la red social en términos de sus dimensiones estructurales y funcionales y el apoyo social percibido, referido a la valoración subjetiva que una persona hace de su red en términos de disposición y satisfacción (Barrón y Sánchez, 2001). Pese a la relevancia de ambos tipos de apoyo, este trabajo se centra en el apoyo social percibido, por su mayor relación con el ajuste psicológico adolescente (Cheung y Sim, 2017).

En cuanto a las fuentes o ámbitos de apoyo, los estudios desarrollados en la infancia y adolescencia tienden a considerar siempre a los progenitores y las amistades como los principales proveedores de apoyo (Simoni y Bauldry, 2018). Además, conviene señalar que, frente a la consideración tradicional de la adolescencia como un periodo vital en el que la familia pierde relevancia frente a las amistades o el grupo de pares, la evidencia constata que la percepción de apoyo parental sigue resultando un importante factor para el ajuste del adolescente (Rodríguez-Fernández, Antonio-Agirre, Ramos-Díaz, y Revuelta, 2020; Rodríguez-Fernández, Ramos-Díaz, Madariaga, Arrivillaga, y Galende, 2016; Watson, Grossman, y Russell, 2019). Así, podría considerarse que la función de apoyo que cumplen ambas fuentes se vuelve más específica (Watson et al., 2019) e incide de manera diferencial sobre las distintas variables del bienestar en los y las adolescentes (Guan y Fuligni, 2015). De tal modo que, los progenitores continuarían siendo una fuente de apoyo o base segura a la que recurrir ante problemas de mayor relevancia y aspectos relacionados con el futuro y la toma de decisiones (Zhu, Tse, Cheung, y Oyserman, 2014), mientras que los pares permitirían cubrir las necesidades afiliativas e interpersonales (Cheung y Sim, 2017).

Por otro lado, en esta época del desarrollo los y las adolescentes confieren cada vez más importancia a la presencia y apoyo de otras personas significativas, como las parejas o las amistades íntimas (Watson et al., 2019) a las que recurren en mayor medida ante el desasosiego o necesidad de proximidad emocional (Guan y Fuligni, 2015) y cuyo apoyo, por tanto, se asocia fuertemente al bienestar (Zhu et al., 2014).

No obstante, resulta importante estudiar el apoyo social percibido en función del sexo y la edad adolescente, ya que se ha constatado que dicha percepción varía en 
relación a dichas variables. De hecho, el sexo condiciona una socialización diferente en el caso de las y los jóvenes, incidiendo en la manera en que se percibe y manifiesta dicho apoyo (Matud, Carballeira, López, Marrero, e Ibáñez, 2002). Igualmente, en función de la edad, las necesidades relacionales de los y las adolescentes cambian (Gaete, 2015), repercutiendo sobre el apoyo social percibido.

Considerando el sexo, se ha observado que las chicas están más preocupadas por la calidad de sus relaciones interpersonales (Demaray y Malecki, 2003; Zach, Yazdi-Ugav, y Zeev, 2016) y tienden a valorar en mayor medida que los chicos el apoyo social externo a la familia (amistades, pares, profesorado, etc.). Investigaciones previas han constatado, también, que las chicas tienden a mencionar a más personas significativas que los chicos y a considerar a estas más relevantes (Guan y Fuligni, 2015).

En la misma dirección apuntan los estudios que observan que las chicas se orientan en mayor medida hacia las amistades y los pares, mientras que los chicos, especialmente jóvenes, parecen recabar apoyo en mayor medida de sus progenitores, más que de sus amistades, percibiendo también un apoyo familiar más alto (Norris y Ayres, 2016). No obstante, a pesar de que las chicas parecen mostrar más importancia y orientación hacia las relaciones externas a la familia, se observa que, también ellas siguen percibiendo a sus progenitores como una fuente de apoyo disponible (Cheung y Sim, 2017).

En lo que respecta a la edad, es reseñable que la percepción de apoyo familiar es más elevada al inicio de la adolescencia que en la adolescencia media (Sanz, 2018), algo que concuerda con el hecho de que, hacia el final de la adolescencia (16-18 años) los y las jóvenes tiendan a preferir pasar cada vez más tiempo con sus pares que con sus progenitores (Nickerson y Nagle, 2005). Se precisa, por tanto, una gradual madurez para que los pares se conviertan en una verdadera fuente de apoyo emocional recíproca (Bokhorst, Sumter, y Westenberg, 2010).

Por otro lado, en relación a la inteligencia emocional cabe señalar que esta se ha clasificado en dos tipos de modelos (Mayer, 2001); el de habilidad (i.e., IE habilidad) y los mixtos (i.e., IE mixta).

El modelo de habilidad define la IE como la destreza en el procesamiento de la información con contenido emocional, describiéndola como "la habilidad para percibir, valorar y expresar la emoción adecuada y adaptativamente; la habilidad para comprender la emoción y el conocimiento emocional; la habilidad para acceder y/o generar sentimientos que faciliten las actividades cognitivas y la acción adaptativa; y, la habilidad para regular las emociones en uno mismo y en otros para promover el crecimiento emocional e intelectual (Mayer y Salovey, 1997, p. 10).

Los modelos mixtos, en cambio, definen la IE como un conjunto de rasgos estables de personalidad, competencias socio-emocionales, aspectos motivacionales y 
diversas habilidades cognitivas. Bar-On (2006) define la Inteligencia Emocional-Social (IES) como un conjunto interrelacionado de competencias, habilidades y facilitadores emocionales y sociales que influyen en la habilidad propia de tener éxito al afrontar las demandas y presiones del medio ambiente.

La toma en consideración de ambos modelos resulta especialmente relevante a nivel teórico, más aún en su relación con el apoyo social, ya que permitiría conocer qué aspectos de la IE, habilidades de tipo cognitivo o rasgos, se asocian al apoyo social percibido en la adolescencia, lo que podría facilitar futuras intervenciones en el ámbito educativo. No obstante, habitualmente los estudios que analizan la relación entre el apoyo social y la IE con la población adolescente se centran en la capacidad explicativa de la IE sobre el apoyo social (Hessel, Loeb, Szwedo, y Allen, 2015) o consideran la incidencia de la IE sobre terceras variables, incluyendo el apoyo social como variable mediadora; asumiendo, por tanto, que serían las habilidades o capacidades emocionales de los y las adolescentes las que facilitarían una mayor percepción de apoyo social (Ye, Yeung, Liu, y Rochelle, 2019).

Sin embargo, son escasos los estudios que analizan la capacidad predictiva del apoyo social sobre la IE (Azpiazu, Esnaola, y Sarasa, 2015), a pesar de que el apoyo social ha mostrado una importante relación con el bienestar psicológico del adolescente (Huang, Costeines, Kaufman, y Ayala, 2014). Se ha constatado, por ejemplo, que adolescentes con elevadas puntuaciones en la percepción del apoyo familiar y el apoyo de amistades muestran una mayor capacidad de resiliencia, afrontamiento de situaciones estresantes y adaptación ante situaciones adversas, así como mejores habilidades para gestionar las emociones de forma positiva (Rodríguez-Fernández, Ramos-Díaz, Ros, y Fernández-Zabala, 2015). Incluso, distintos estudios confirman la relación entre el apoyo social percibido y diversas medidas de IE. Por ejemplo, se ha comprobado esta correlación entre la percepción de disponibilidad de apoyo social material e inmaterial (emocional) y medidas de IE evaluadas con test de habilidad (Zeidner, Matthews, y Olenick, 2016), el apoyo percibido de la familia, los pares y el profesorado y medidas de IE habilidad utilizando autoinformes (Atoum y Al-Shoboul, 2018) así como siguiendo un modelo mixto de IE medido con autoinforme (Kong, Zhao, y You, 2012).

Se considera que el apoyo proporcionado por la familia y las amistades contribuye a las habilidades emocionales (Fernández-Berrocal y Ramos, 2016; Fernández-Lasarte, Ramos-Díaz, y Axpe, 2019) y desde la perspectiva de la IE como habilidad, un estudio con adolescentes (entre 11 y 18 años) constata que, tanto en el caso de los chicos como de las chicas, el apoyo familiar predice las puntuaciones en las dimensiones de claridad y reparación, mientras el apoyo de las amistades predeciría la atención, la claridad y la reparación emocional (Azpiazu et al., 2015). Siguiendo un modelo similar, Atoum y Al-Shoboul (2018) encuentran que, entre adolescentes de 14-15 años, el apoyo emocional de la familia (especialmente) y las amistades (en mucha 
menor medida), explican la conciencia sobre las propias emociones, así como el uso y la regulación emocional propia y de las demás personas.

Sin embargo, hasta ahora no se conocen estudios que analicen el papel predictivo del apoyo social percibido sobre la IE desde el modelo mixto entre adolescentes de contextos latinoamericanos. Es por ello, que el objetivo de este trabajo es analizar la capacidad predictiva del apoyo social percibido (de las personas significativas, la familia y las amistades) sobre la IE habilidad (considerando las dimensiones de atención, claridad y reparación emocional) y la IE mixta (teniendo en cuenta las dimensiones de habilidad intrapersonal, habilidad interpersonal, el manejo del estrés y la habilidad de adaptación) considerando por separado el sexo y la edad adolescente, ya que se ha observado que estas dos variables condicionan la autopercepción en relación a aspectos del bienestar adolescente (Maganto, Péris, y Sánchez, 2019). El uso de los dos modelos de IE (de habilidad y mixto) permitirá conocer en qué medida el apoyo social incide sobre las habilidades, más de tipo cognitivo o de tipo mixto, de la IE de los y las adolescente, lo que podría facilitar, a nivel práctico, el diseño de intervenciones que contribuyesen a la mejora de una variable tan relevante en la adolescencia.

\section{MÉTODO}

\section{Participantes}

En este estudio participaron 490 estudiantes de enseñanza secundaria (de $1^{\circ}$ hasta $\left.6^{\circ}\right), 300$ chicas $(61.2 \%)$ y 190 chicos $(38.8 \%)$ seleccionados aleatoriamente de centros públicos (6) y privados (2), residentes de la República Dominicana. La muestra se dividió en dos submuestras, adolescencia temprana 11-14 años $(M=12.49$, $D T=.615)$ y adolescencia media, $15-19$ años $(M=15.06, D T=.811)$.

\section{Instrumentos}

Para evaluar el apoyo social percibido se utilizó el cuestionario Multidimensional Scale of Perceived Social Support (MSPSS) de Zimet, Dahlem, Zimet, y Farley (1988), traducido al español y validado por Arechavala y Miranda (2002). La escala MSPSS está compuesta por 12 ítems y evalúa la percepción que tienen las personas del apoyo social informal con el que cuentan, recogiendo información del apoyo social percibido por los individuos en tres áreas: la familia, las amistades y los otros significativos o personas significativas. Los ítems se valoran empleando una escala de respuestas que oscilan entre 1 (totalmente en desacuerdo) y 7 (totalmente de acuerdo). Esta escala ha mostrado tener una buena consistencia interna en sus tres subescalas: familia $(\alpha=.80)$, amistades $(\alpha=.84)$ y otros significativos $(\alpha=.80)$.

La inteligencia emocional se valoró mediante dos cuestionarios: (1) Trait Meta Mood Scale (TMMS) de Salovey y Mayer (1990), adaptado y validado al 
castellano por Fernández-Berrocal, Extremera, y Ramos (2004). Se trata de una escala de 24 ítems agrupados en tres dimensiones: atención emocional, claridad emocional y reparación emocional. La consistencia interna de cada dimensión en este estudio fue de .78, .75 y .77 respectivamente y; (2) Emotional Quotient Inventory: Youth Version Short (EQ-i: YV-S: Bar-On y Parker, 2000), traducido por Caraballo y Villegas (2001). Este autoinforme está diseñado para medir la IE de los/as niños/as y adolescentes entre los 7 y 18 años a través de 30 ítems que recogen las dimensiones de: habilidad intrapersonal, habilidad interpersonal, manejo del estrés y habilidad de adaptación. La consistencia interna (alpha de Cronbach) obtenida en este estudio en las diferentes subescalas se indica a continuación: intrapersonal $(\alpha=.52)$, interpersonal $(\alpha=.70)$, manejo del estrés $(\alpha=.79)$ y adaptabilidad $(\alpha=.75)$.

\section{Procedimiento}

Para desarrollar esta investigación se solicitó el permiso a las autoridades del Ministerio de Educación de la República Dominicana. Posteriormente, se contactó con los centros de educación secundaria para exponerles los objetivos del estudio y describirles los instrumentos de evaluación. Se convocó una reunión con las familias del alumnado para explicarles el estudio y, a la vez, solicitar el consentimiento para que sus hijos e hijas participaran en la investigación. Se seleccionaron ocho centros educativos de forma aleatoria, seis públicos y dos privados, los cuales manifestaron durante todo el proceso su disposición a colaborar. Se aplicaron las pruebas de forma grupal y a los y las discentes se les proporcionaron todas las explicaciones e instrucciones pertinentes.

\section{Análisis de datos}

El tratamiento estadístico de los datos se realizó con el Software SPSS Statistics en su versión 23 para Windows. Para el análisis de la consistencia interna de los cuestionarios se utilizó el alfa de Cronbach y para el análisis de la capacidad predictiva del apoyo social percibido en la inteligencia emocional se llevó a cabo una regresión lineal múltiple.

\section{RESULTADOS}

A continuación, se muestran los resultados obtenidos al analizar la capacidad explicativa de las tres fuentes de apoyo social (personas significativas, familia y amistades) sobre las dimensiones de la IE concebida como habilidad (ver Tabla 1).

En el grupo de chicas más jóvenes (11 a 14 años) se observa que el apoyo social percibido por parte de las personas significativas permite explicar parte de la varianza en atención emocional, aproximadamente un $13 \%\left(R^{2}=.128 ; \beta=.446\right.$, $p \leq .001$ ), algo que no ocurre entre las chicas de más edad, para las que ninguna de las 
fuentes de apoyo social muestra capacidad explicativa significativa. En el caso de la claridad emocional, sólo el apoyo percibido de las amistades permite explicar parte de su varianza entre las chicas mayores (15-19 años), casi un $15 \%\left(R^{2}=.147 ; \beta=.273\right.$, $p \leq .010$ ), no mostrando capacidad explicativa entre las chicas más jóvenes. Sólo en la dimensión de reparación emocional existe cierta coincidencia entre los dos grupos de edad. En ambos casos, percibir apoyo familiar permite explicar parte de la varianza, siendo la capacidad explicativa ligeramente superior entre las chicas más jóvenes $(\beta=.221, p \leq .05)$ que entre las mayores $(\beta=.200, p \leq .05)$, ya que entre ellas el apoyo percibido de las amistades también permite explicar parte de las puntuaciones obtenidas en reparación emocional $(\beta=.320, p \leq .001)$.

Tabla 1. Capacidad predictiva del Apoyo Social sobre la IE de las chicas (modelo de habilidad)

\begin{tabular}{|c|c|c|c|c|c|c|c|c|c|c|c|c|c|}
\hline \multirow{6}{*}{ 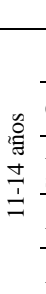 } & & \multicolumn{4}{|c|}{ Atención } & \multicolumn{4}{|c|}{ Claridad } & \multicolumn{4}{|c|}{ Reparación } \\
\hline & & $R^{2}$ & $\beta$ & $t$ & $p$ & $R^{2}$ & $\beta$ & $t$ & $p$ & $R^{2}$ & $\beta$ & $t$ & $p$ \\
\hline & Constante & \multirow{4}{*}{.128} & - & 2.49 & .015 & \multirow{4}{*}{.025} & - & 2.76 & .007 & \multirow{4}{*}{.127} & - & 1.79 & .077 \\
\hline & $\begin{array}{l}\text { Apoyo de personas } \\
\text { significativas }\end{array}$ & & .446 & 3.78 & $.000^{* k * k *}$ & & .143 & 1.14 & .256 & & .131 & 1.11 & .271 \\
\hline & Apoyo familiar & & -.070 & -.628 & .532 & & .145 & 1.24 & .220 & & .221 & 1.99 & $.050^{*}$ \\
\hline & Apoyo amistades & & -.117 & -1.04 & .302 & & .036 & .301 & .764 & & .203 & 1.80 & .075 \\
\hline \multirow{4}{*}{ 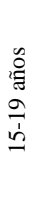 } & Constante & \multirow{4}{*}{.050} & - & 8.26 & .000 & \multirow{4}{*}{.147} & - & 4.81 & .000 & \multirow{4}{*}{.153} & - & 6.38 & .000 \\
\hline & $\begin{array}{l}\text { Apoyo de personas } \\
\text { significativas }\end{array}$ & & .117 & 1.16 & .249 & & .084 & .883 & .379 & & -.064 & -.672 & .502 \\
\hline & Apoyo familiar & & -.016 & -.183 & .855 & & .115 & 1.38 & .170 & & .200 & 2.41 & $.017^{*}$ \\
\hline & Apoyo amistades & & .139 & 1.43 & .156 & & .273 & 2.97 & $.003^{* *}$ & & .320 & 3.50 & $.001^{* * 0 \times k}$ \\
\hline
\end{tabular}

En lo que respecta a la capacidad explicativa del apoyo social sobre la IE concebida desde un modelo mixto, los resultados cambian ligeramente (ver Tabla 2).

Cuando la IE se concibe desde un modelo mixto, las fuentes de apoyo social pierden relevancia como factor explicativo entre las chicas de más edad. De hecho, se constata que para las chicas más jóvenes, el apoyo de las personas significativas permite explicar un mayor porcentaje de la varianza de la dimensión interpersonal $\left(R^{2}=.212\right.$; $\beta=.518, p \leq .001)$ que entre las chicas mayores $\left(R^{2}=.085 ; \beta=.308, p \leq .010\right)$. También es el apoyo de las personas significativas el que permite explicar parte de la varianza en adaptabilidad de las chicas más jóvenes $\left(R^{2}=.086 ; \beta=.245,=p \leq .05\right)$, sin alcanzar capacidad explicativa significativa entre las chicas mayores. En el manejo del estrés $\left(R^{2}=.078\right)$ entre las chicas más jóvenes el apoyo de las personas significativas explica menores puntuaciones $(\beta=-.279, p \leq .05)$, mientras que la percepción del apoyo de amistades muestra puntuaciones superiores $(\beta=.302, p \leq .010)$. Entre las chicas de más edad sólo el apoyo familiar explica, muy ligeramente, el manejo del estrés $\left(R^{2}=.013\right.$; 
$\beta=.199, p \leq .05)$. La única dimensión en la que se observa coincidencia entre ambos grupos de edad es la intrapersonal, en la que el apoyo social de las amistades permite predecir, especialmente en el caso de las chicas mayores $\left(R^{2}=.103 ; \beta=.348, p \leq .001\right.$ frente a $\left.R^{2}=.039 ; \beta=.275, p \leq .05\right)$, mejores puntuaciones en la escala.

Tabla 2. Capacidad predictiva del Apoyo Social sobre la IE de las chicas (modelo mixto)

\begin{tabular}{|c|c|c|c|c|c|c|c|c|c|c|c|c|c|c|c|c|}
\hline & & \multicolumn{4}{|c|}{ Intrapersonal } & \multicolumn{4}{|c|}{ Interpersonal } & \multicolumn{4}{|c|}{ Manejo estrés } & \multicolumn{3}{|c|}{ Adaptabilidad } \\
\hline & & $R^{2}$ & $\beta$ & $t$ & $p$ & $R^{2}$ & $\beta$ & $t$ & $p$ & $R^{2}$ & $\beta$ & $t$ & $p$ & $R^{2}$ & $\beta$ & $p$ \\
\hline \multirow{2}{*}{$\begin{array}{l}\mathscr{8} \\
\stackrel{\Xi}{\Xi} \\
\pm \\
\stackrel{1}{ \pm} \\
=\end{array}$} & $\begin{array}{l}\text { Apoyo de personas } \\
\text { significativas }\end{array}$ & \multirow{2}{*}{.039} & .014 & -.110 & .913 & \multirow{2}{*}{.212} & .518 & 4.62 & $.000^{* 0 *}$ & \multirow{2}{*}{.078} & -.279 & -2.30 & $.024^{*}$ & -086 & .245 & $2.03 .046^{*}$ \\
\hline & Apoyo amistades & & .275 & 2.33 & $.022^{*}$ & & -.068 & -.640 & .524 & & .302 & 2.61 & $.011^{* *}$ & & .180 & 1.56 .123 \\
\hline \multirow{3}{*}{ 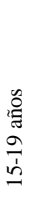 } & Constante & & - & 6.75 & .000 & & - & 10.16 & .000 & \multirow{3}{*}{.013} & - & 7.57 & .000 & \multirow{2}{*}{.040} & - & 7.84 .000 \\
\hline & $\begin{array}{l}\text { Apoyo de personas } \\
\text { significativas }\end{array}$ & 103 & -.148 & -1.52 & .131 & 085 & .308 & 3.14 & $.002^{* *}$ & & -.088 & -.864 & .389 & & .155 & 1.53 .129 \\
\hline & Apoyo amistades & & .348 & 3.70 & $.000^{6 * *}$ & & .058 & .609 & .543 & & .002 & .024 & .981 & & .072 & .732 .465 \\
\hline
\end{tabular}

Nota. ${ }^{* * *} p \leq .001,{ }^{* *} p \leq .010,{ }^{*} p \leq .05$.

Los resultados obtenidos con el grupo de chicos difieren en gran medida de los observados entre las chicas. Considerando la IE desde un modelo de habilidad (ver Tabla 3), se constata que únicamente el apoyo social percibido por parte de la familia refleja cierta capacidad explicativa de las puntuaciones en reparación entre los chicos más jóvenes $\left(R^{2}=.164 ; \beta=.633, p \leq .010\right)$.

Tabla 3. Capacidad predictiva del Apoyo Social sobre la IE de los chicos (modelo de habilidad)

\begin{tabular}{|c|c|c|c|c|c|c|c|c|c|c|c|c|c|}
\hline & & \multicolumn{4}{|c|}{ Atención } & \multicolumn{4}{|c|}{ Claridad } & \multicolumn{4}{|c|}{ Reparación } \\
\hline & & $R^{2}$ & $\beta$ & $t$ & $p$ & $R^{2}$ & $\beta$ & $t$ & $p$ & $R^{2}$ & $\beta$ & $t$ & $p$ \\
\hline & Constante & \multirow{4}{*}{.003} & - & 4.75 & .000 & \multirow{4}{*}{.035} & - & 4.48 & .000 & \multirow{4}{*}{.164} & - & 4.53 & .000 \\
\hline \multirow{3}{*}{$\begin{array}{l}\text { 志 } \\
\text { 士 } \\
\text { I } \\
=\end{array}$} & $\begin{array}{l}\text { Apoyo de personas } \\
\text { significativas }\end{array}$ & & -.074 & -.310 & .758 & & .001 & .004 & .997 & & .001 & .004 & .997 \\
\hline & Apoyo familiar & & .275 & 1.22 & .229 & & .339 & 1.54 & .132 & & .633 & 3.08 & .004 \\
\hline & Apoyo amistades & & .026 & .116 & .908 & & -.037 & -.166 & .869 & & .413 & -2.00 & .052 \\
\hline & Constante & \multirow{4}{*}{.033} & - & 8.82 & .000 & \multirow{4}{*}{.006} & - & 7.51 & .000 & \multirow{4}{*}{.046} & - & 8.09 & .000 \\
\hline & $\begin{array}{l}\text { Apoyo de personas } \\
\text { significativas }\end{array}$ & & -.014 & -.106 & .916 & & .092 & .673 & .502 & & .239 & 1.79 & .076 \\
\hline 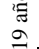 & Apoyo familiar & & -.117 & -.930 & .355 & & .011 & .086 & .932 & & .099 & .808 & .421 \\
\hline 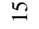 & Apoyo amistades & & .219 & 1.73 & .086 & & .104 & .823 & .412 & & -.074 & -0.60 & .552 \\
\hline
\end{tabular}


Cuando se considera la capacidad explicativa del apoyo social sobre la IE concebida desde un modelo mixto, los resultados entre los chicos vuelven a mostrar que únicamente el apoyo familiar y sólo entre los adolescentes más jóvenes, explica parte de la varianza de la IE (ver Tabla 4).

Tabla 4. Capacidad predictiva del Apoyo Social sobre la IE de los chicos (modelo mixto)

\begin{tabular}{|c|c|c|c|c|c|c|c|c|c|c|c|c|c|c|c|c|}
\hline & \multicolumn{4}{|c|}{ Intrapersonal } & \multicolumn{4}{|c|}{ Interpersonal } & \multicolumn{4}{|c|}{ Manejo estrés } & \multicolumn{4}{|c|}{ Adaptabilidad } \\
\hline & $R^{2}$ & $\beta$ & $t$ & $p$ & $R^{2}$ & $\beta$ & $t$ & $p$ & $R^{2}$ & $\beta$ & $t$ & $p$ & $R^{2}$ & $\beta$ & $t$ & $p$ \\
\hline Constante & \multirow{4}{*}{.019} & - & 7.12 & .000 & \multirow{4}{*}{.168} & - & 4.16 & .000 & \multirow{4}{*}{. 043} & - & 5.34 & .000 & \multirow{4}{*}{.058} & - & 5.60 & .000 \\
\hline $\begin{array}{l}\text { Apoyo de personas } \\
\text { significativas }\end{array}$ & & .093 & .383 & .703 & & .047 & .218 & .828 & & .239 & 1.03 & .307 & & -.298 & -1.30 & .202 \\
\hline '胥 Apoyo familiar & & .117 & .511 & .612 & & .436 & 2.13 & $.039^{*}$ & & -.426 & -1.94 & .059 & & .443 & 2.03 & .048 \\
\hline$=$ Apoyo amistades & & -.190 & -.823 & .415 & & .003 & .014 & .989 & & .277 & 1.25 & .217 & & .087 & .398 & .692 \\
\hline Constante & \multirow{4}{*}{.020} & - & 8.57 & .000 & \multirow{4}{*}{.027} & - & 9.32 & .000 & \multirow{4}{*}{.006} & - & 8.40 & .000 & \multirow{4}{*}{.005} & - & 9.19 & .000 \\
\hline $\begin{array}{l}\text { Apoyo de personas } \\
\text { significativas }\end{array}$ & & .043 & .312 & .755 & & .094 & .699 & .486 & & -.033 & -.242 & .810 & & .072 & .521 & .604 \\
\hline 胥Apoyo familiar & & -.132 & -1.04 & .300 & & -.148 & -1.19 & .236 & & 215 & 1.71 & .090 & & .002 & .017 & .987 \\
\hline Apoyo amistades & & .143 & 1.13 & .262 & & .222 & 1.78 & .079 & & -.036 & -.283 & .778 & & -.030 & -.236 & .814 \\
\hline
\end{tabular}

Concretamente, el apoyo de la familia alcanza a explicar parte de las puntuaciones en las dimensiones de adaptabilidad $\left(\mathrm{R}^{2}=.058 ; \beta=.443, p \leq .05\right) \mathrm{e}$ interpersonal $\left(\mathrm{R}^{2}=.168 ; \beta=.436, p \leq .05\right)$ de los chicos más jóvenes, sin llegar a observarse ninguna otra fuente de apoyo con capacidad explicativa significativa.

\section{DISCUSIÓN Y CONCLUSIONES}

El objetivo del presente estudio consistía en analizar la capacidad predictiva del apoyo social percibido (de las personas significativas, la familia y las amistades) sobre la IE. En general, los resultados sugieren que el apoyo social es claramente más relevante entre las chicas que entre los chicos a la hora de predecir la IE desde ambos modelos, tanto en la adolescencia temprana como en la adolescencia media. Estos resultados no coinciden con estudios previos (Azpiazu et al., 2015), en los que el apoyo social era significativo para ambos sexos. Quizá los presentes resultados puedan explicarse por una socialización diferencial que educa a los hombres más hacia la independencia, la producción y lo material-instrumental y sin embargo, dirige a las mujeres hacia lo emocional, la relación y el cuidado (Suberviola, 2020).

Por otro lado, la capacidad predictiva del apoyo familiar en la regulación y reparación de las emociones ha sido significativa en la adolescencia temprana en ambos sexos y en la adolescencia media entre las chicas, coincidiendo con estudios previos (Atoum y Al-Shoboud, 2018; Azpiazu et al., 2015) según los cuales un buen apoyo 
familiar es un factor clave para manejar el estrés de forma positiva y para el desarrollo de habilidades emocionales (Fernández-Berrocal y Ramos, 2016). Este resultado confirma la importancia que la familia sigue teniendo en la adolescencia (Cheung y Sim, 2017; Watson et al., 2019; Zhu et al., 2014), a pesar de la necesidad de los y las adolescentes de desarrollar una mayor independencia y la importancia que adquieren las amistades, la cual aumenta especialmente hacia el final del periodo (Nickerson y Nagle, 2005), constituyéndose como mayor fuente de apoyo (Bokhorst et al., 2010).

En este sentido, los resultados aquí obtenidos concordarían con la mayor importancia de las relaciones sociales y el apoyo social extrafamiliar, de las amistades y de otras personas significativas (Cheung y Sim, 2017; Guan y Fuligni, 2015) para las mujeres, ya que el apoyo de las amistades sólo ha sido significativo entre las chicas (prediciendo las dimensiones de reparación, intrapersonal y manejo del estrés en la adolescencia temprana y claridad, reparación e intrapersonal en la adolescencia media). Entre los chicos el apoyo de las amistades no ha sido significativo en ninguno de los casos. Estos resultados contrastan con los de trabajos previos, pues Azpiazu et al. (2015) encontraron que el apoyo de las amistades predecía la atención, la claridad y la reparación emocional en ambos sexos. No obstante, apuntan en la línea de otros estudios en los que el apoyo de las amistades muestra un mayor valor como apoyo emocional para las chicas (Teoh, Chong, Yip, Lee, y Wong, 2015), mientras que entre los chicos las amistades suelen tener una función más instrumental o de compañeros para la realización de actividades conjuntas.

Asimismo, destaca la importancia, en el modelo mixto de la IE, del apoyo proporcionado por las personas significativas entre las chicas en la adolescencia temprana, ya que predice las dimensiones interpersonal, manejo del estrés y la adaptabilidad. Es decir, parece que, además de la familia y las amistades, pueden existir otras personas significativas que tienen importancia en la IE de las adolescentes más jóvenes.

En referencia a la valoración y resultados de los modelos de IE, los resultados obtenidos en este trabajo apuntan a una mayor capacidad predictiva del apoyo social sobre la IE habilidad que sobre la IE mixta, cuestión que podría tener gran relevancia en el diseño de intervenciones que puedan mejorar dos activos tan importantes como son el apoyo social y la IE en la adolescencia.

No obstante, es preciso señalar que los resultados obtenidos en esta investigación deben considerarse con cautela, debido a ciertas limitaciones de la misma, como la poca consistencia interna obtenida en la dimensión intrapersonal. Además, se han utilizado dos instrumentos de autoinforme para medir la IE desde el modelo de habilidad y desde el modelo mixto; por tanto, sería interesante que estudios posteriores empleasen también test de ejecución, así como otras medidas de autoinforme. Igualmente, sería necesario ampliar la muestra adolescente para analizar la adolescencia 
tardía. Asimismo, sería oportuno analizar la capacidad predictiva del apoyo social sobre la IE utilizando métodos estadísticos más robustos como los modelos de ecuaciones estructurales.

Más allá de estas limitaciones, este trabajo contribuye a conocer la capacidad predictiva del apoyo social percibido sobre dos modelos de la inteligencia emocional, el modelo de habilidad y el mixto, en la etapa de la adolescencia, considerando por separado el sexo y la edad de los y las adolescentes. En general, los resultados han indicado que el apoyo social tiene mayor capacidad predictiva de la IE entre las chicas, lo que sugiere la necesidad de tener muy en cuenta la red social que poseen las mujeres y potenciar dicho apoyo, desarrollando programas de intervención que persigan el objetivo de mejorar las relaciones entre los, y especialmente, las adolescentes y la familia, entre los iguales, e incluso entre el alumnado y el profesorado, ya que también pueden ser personas significativas en la etapa adolescente.

\section{Agradecimientos}

Este trabajo ha recibido financiación del Ministerio de Educación Superior, Ciencia y Tecnología (MESCyT), República Dominicana. Por otra parte, se enmarca dentro del trabajo realizado en el Grupo Consolidado de Investigación del Sistema Universitario Vasco IT934-16, así como del proyecto de investigación PPG17/61 de la Universidad del País Vasco y del proyecto EDU2017-83949-P del subprograma estatal de Generación del Conocimiento del Ministerio de Economía, Industria y Competitividad.

\section{REFERENCIAS}

Arechabala, M.C., y Miranda, C. (2002). Validación de una escala de apoyo social percibido en un grupo de adultos mayores adscritos a un programa de hipertensión de la región metropolitana. Ciencia y Enfermería, 8(1), 49-55.

Atoum, A.Y., y Al-Shoboul, R.A. (2018). Emotional support and its relationship to Emotional intelligence. Advances in Social Sciences Research Journal, 5(1), 7-16. doi:10.14738/assrj.51.4095

Azpiazu, L., Esnaola, I., y Sarasa, M. (2015). Capacidad predictiva del apoyo social en la inteligencia emocional de adolescentes. European Journal of Education and Psychology, 8(1), 23-29. doi:10.1016/j.ejeps.2015.10.003

Bar-On, R. (2006). The Bar-On model of emotional-social intelligence (ESI). Psicothema, 18, 13-25.

Bar-On, R., y Parker, J. (2000). The Bar-On Emotional Quotient Inventory: Youth Version (EQ-i: YV). Technical Manual (Traducido al español por C. M. Caraballo y O. Villegas). Toronto, Canada: Multi-Health Systems, Inc.

Barrón, A., y Sánchez, E. (2001). Estructura social, apoyo social y salud mental. Psicothema, 13(1), 17-23.

Bokhorst, C.L., Sumter, S.R., y Westenberg, P.M. (2010). Social support from parents, friends, classmates, and teachers in children and adolescents aged 9 to 18 years: Who is 
JIMÉNEZ, AXPE, y ESNAOLA. El rol del apoyo social percibido en la predicción de la IE

perceived as most supportive? Social Development, 19(2), 417-426. doi:10.1111/j.1467-9507.2009.00540.x

Cheung, H.S., y Sim, T.N. (2017). Social support from parents and friends for Chinese adolescents in Singapore. Youth \& Society, 49(4), 548-564. doi:10.1177/0044118X14559502

Demaray, M.K., y Malecki, C.K. (2003). Importance ratings of socially supportive behaviors by children and adolescents. School Psychology Review, 32(1), 108- 131.

Fernández-Berrocal, P., Extremera, N., y Ramos, N. (2004). Validity and reliability of the Spanish modified version of the Trait Meta-Mood Scale. Psychological Reports, 94(3), 751-755. doi:10.2466/pr0.94.3.751-755

Fernández-Berrocal, P., y Ramos, N.D. (2016). Desarrolla tu inteligencia emocional. Barcelona: Kairós.

Fernández-Lasarte, O., Ramos-Díaz, E., y Axpe, I. (2019). Rendimiento académico, apoyo social percibido e inteligencia emocional en la universidad. European Journal of Investigation in Health, Psychology and Education, 9(1), 39-49. doi:10.30552/ejihpe.v9i1.315

Gaete, V. (2015). Desarrollo psicosocial del adolescente. Revista Chilena de Pediatría, 86(6), 436-443.

Guan, S.S.A., y Fuligni, A.J. (2015). Changes in parent, sibling, and peer support during the transition to young adulthood. Journal of Research on Adolescence, 26, 286-299. doi:10.1111/jora.12191

Hessel, E.T., Loeb, E.L., Szwedo, D.E., y Allen, J.P. (2015). Predictions from early adolescent emotional repair abilities to functioning in future relationships. Journal of Research on Adolescence, 26(4), 776-789. doi:10.1111/jora.12229

Huang, C.Y., Costeines, J., Kaufman, J.S., y Ayala, C. (2014). Parenting stress, social support, and depression for ethnic minority adolescent mothers: Impact on child development. Journal of Child and Family Studies, 23(2), 255-262. doi:10.1007/s10826-013-9807-1

Kong, F., Zhao, J., y You, X. (2012). Social support mediates the impact of emotional intelligence on mental distress and life satisfaction in Chinese young adults. Personality and Individual Differences, 53(4), 513-517. doi:10.1016/j.paid.2012.04.021

Lin, N., y Ensel, W. (1989). Life stress and health: Stressors and resources. American Sociological Review, 54, 382-399. doi: 10.2307/209561

Maganto, C., Péris, M., y Sánchez, R. (2019). El bienestar psicológico en la adolescencia: Variables psicológicas asociadas y predictoras. European Journal of Education and Psychology, 12(2), 139-151. doi:10.30552/ ejep.v12i2.279

Matud, P., Carballeira, M., López, M., Marrero, R., e Ibañez, I. (2002). Apoyo social y salud: un análisis de género. Salud Mental, 25(2), 32-37.

Mayer, J.D. (2001). Emotion, intelligence, and emotional intelligence. En J.P. Forgas (Ed.), The handbook of affect and social cognition (pp. 410-431). Mahwah, New Jersey: Lawrence Erlbaum \& Associates.

Mayer, J.D., y Salovey, P. (1997). What is emotional intelligence? En P. Salovey y D. Sluyter (Eds.), Emotional development and emotional intelligence: Implications for educators (pp. 3-34). New York: Basic Books.

Nickerson, A.B., y Nagle, R.J. (2005). Parent and peer attachment in late childhood and early adolescence. Journal of Early Adolescence, 25, 223-249. doi:10.1177/0272431604274174

Norris, S.M., y Ayres, C.G. (2016). Factors influencing the health promoting physical activity behaviors of diverse urban adolescents. Journal of Nursing Practice Applications $y$ Reviews of Research, 6(1), 16-23. 
Pérez-Escoda, N., Filella, G., Alegre, A., y Bisquerra, R. (2018). Desarrollo de la competencia emocional de maestros y alumnos en contextos escolares. Electronic Journal of Research in Education Psychology, 10(28), 1183-1208.

Rodríguez-Fernández, A., Antonio-Agirre, I., Ramos-Díaz, E., y Revuelta, L. (2020). The role of affect-communication and rule setting in perceived family support and school adjustment. European Journal of Education and Psychology,13(1), 207-220. doi:10.30552/ejep.v13i1.288

Rodríguez-Fernández, A., Ramos-Díaz, E., Madariaga, J. M., Arrivillaga, A., y Galende, N. (2016). Steps in the construction and verification of an explanatory model of psychosocial adjustment. European Journal of Education and Psychology, 9(1), 20-28. doi:10.1016/j.ejeps.2015.11.002

Rodríguez-Fernández, A., Ramos-Díaz, E., Ros, I., y Fernández-Zabala, A. (2015). Relaciones de la resiliencia con el autoconcepto y el apoyo social percibido en una muestra de adolescentes. Acción Psicológica, 12(2), 1-14. doi:10.5944/ap.12.2.14903

Salavera, C., Usán, P., y Teruel, P. (2019). The relationship of internalizing problems with emotional intelligence and social skills in secondary education students: gender differences. Psicologia: Reflexão e Crítica, 32(1), 2-9. doi:10.1186/s41155-018-0115-y

Salovey, P., y Mayer, J.D. (1990). Emotional intelligence. Imagination, Cognition and Personality, 9(3), 185-211.

Sanz, D.M. (2018). Niveles de actividad física y apoyo social percibido del alumnado adolescente de la provincia de Soria (Tesis doctoral). Universidad Complutense de Madrid: España.

Simoni, Z. R., y Bauldry, S. (2018). Moving during adolescence and depressive symptoms: The role of social support. Youth and Society, 52(4), 639-660. doi:10.1177/0044118X18757149

Suberviola, I. (2020). La socialización diferencial emocional de género como factor predictor del carácter. IQUAL. Revista de Género e Igualdad, 3, 80-93 doi:10.6018/iqual.369611

Teoh, A.N., Chong, L.X., Yip, C.C. E., Lee, P.S. H., y Wong, J.W.K. (2015). Gender as moderator of the effects of online social support from friends and strangers: A study of Singaporean college students. International Perspectives in Psychology: Research, Practice, Consultation, 4(4), 254-266. doi:10.1037/ipp0000040

Watson, R.J., Grossman, A.H., y Russell, S.T. (2019). Sources of social support and mental health among LGB youth. Youth and Society, 51(1), 30-48. doi:10.1177/0044118X16660110

Ye, J., Yeung, D.Y., Liu, E.S., y Rochelle, T.L. (2019). Sequential mediating effects of provided and received social support on trait emotional intelligence and subjective happiness: A longitudinal examination in Hong Kong Chinese university students. International Journal of Psychology, 54(4), 478-486. doi:10.3389/fpsyg.2015.00395

Zach, S., Yazdi-Ugav, O., y Zeev, A. (2016). Academic achievements, behavioral problems, and loneliness as predictors of social skills among students with and without learning disorders. School Psychology International, 37(4), 378-396. doi:10.1177/0143034316649231

Zeidner, M., Matthews, G., y Olenick, D.S. (2016). Cognitive-social sources of wellbeing: Differentiating the roles of coping style, social support and emotional intelligence. Journal of Happiness $\quad$ Studies, 17(6), 2481-2501. doi:10.1007/s10902-015-9703-z

Zhu, S., Tse, S., Cheung, S.H., y Oyserman, D. (2014). Will I get there? Effects of parental support on children's possible selves. British Journal of Educational Psychology, 84(3), 435-453. doi:10.1111/bjep.12044 
JIMÉNEZ, AXPE, y ESNAOLA. El rol del apoyo social percibido en la predicción de la IE

Zimet, G.D., Dahlem, N.W., Zimet, S.G., y Farley, G.K. (1988). The multidimensional scale of perceived social support. Journal of Personality Assessment,52(1), 30-41. doi:10.1207/s15327752jpa5201_2

Recibido: 31 de mayo de 2019

Recepción Modificaciones: 15 de junio de 2019

Aceptado: 1 de julio de 2019 